\title{
CD200 Expression in Diagnostic and Prognostic Assessment of Mature B Cell Lymphophoproliferative Neoplasms
}

\author{
Neveen Bahaa El Din Fouad ${ }^{1}$, Noha Yehia Ibrahim²*, Rania Salah Abdel Aziz ${ }^{3}$, \\ Sarah Khalid Ibrahim ${ }^{3}$
}

\begin{abstract}
Background: Multiparameter flow cytometry is a useful tool for diagnostic evaluation of mature B-cell neoplasms $(\mathrm{MBN})$. Recently, it has been shown that assessment of CD200 expression may improve the distinction between chronic lymphocytic leukemia (CLL; CD200 positive) and mantle cell lymphoma (MCL; CD200 negative), but any potential as a prognostic marker for CLL remains to be established. Materials and methods: This cross sectional study was conducted on sixty-seven patients newly diagnosed as having mature B-cell lymphoproliferative disorders Levels of CD 200 in lymphoma cells were assessed. Results: CD200 was consistently expressed in CLL and hairy cell leukemia B cells, but not in MCL cells. Heterogeneous expression was noted in other CD5 positive Non-Hodgkin lymphomas. High CD200 expression ( $\geq 50 \%$ ) was associated with a higher CD5, 19 and CD23 expression, older age, higher TLC and absolute lymphocyte count, hepatomegaly, splenomegaly and a higher Rai stage. There were no significant correlations between CD200 expression and response to treatment. Conclusion: CD200 could be of high value in distinguishing CLL, MCL, and atypical CLL. CD200 expression can also be of prognostic and therapeutic value in CLL cases.
\end{abstract}

Keywords: CD200- mature B-cell neoplasms- flowcytometry- CLL

Asian Pac J Cancer Prev, 19 (12), 3383-3392

\section{Introduction}

The diagnosis of mature B cell lymphoproliferative disorders is based on immunophenotyping (IMP) (Van Dongen et al., 2012; Ivancević et al., 2014). Mantle cell lymphoma (MCL) is an aggressive disease in comparison to chronic lymphocytic leukemia (CLL) and requires intensive treatment. Sometimes MCL is misdiagnosed as CLL (Challagundla et al., 2014).

CLL is usually diagnosed by IMP being positive for CD23, having weak cell surface expression immunoglobulin (sIg), lacking expression of FMC and weak expression of CD20. MCL is usually CD23negative and CD20 strongly positive. However, in some cases, CD23 is expressed in MCL and is lacking in CLL leading to misdiagnosis (Barna et al., 2008).

A membrane glycoprotein expressed in all CD19+ B lymphocytes and some of the T is known as CD200. It is not expressed in natural killer cells, monocytes, granulocytes or platelets. This glycoprotein previously named OX2 is also expressed in myeloma plasma cells and acute myeloid leukemia (Moreaux et al., 2008).

Some studies have evaluated the role of CD200 expression in the differential diagnosis of CLL and other mature B cell lymphoproliferative disorders
(Sandes et al., 2013; Eldesoukey et al., 2012) however its correlation with the prognosis and treatment outcome is still not properly investigated.

In this work we studied the role of CD200 expression in the differential diagnosis and prognosis of CLL in relation to other mature $\mathrm{B}$ cell lymphoproliferative disorders.

\section{Materials and Methods}

\section{Patient and methods}

Patient

This study included 67 patients with De Novo Chronic Lymphoproliferative disorders (Mature B-Cell Lymphoproliferative Disorders) who presented to the oncology outpatient clinics of the National Cancer Institute (NCI) - Cairo University during the period from March 2014 to December 2014, in addition to 20 age and sex matched healthy volunteers as control subjects.

Inclusion criteria included all patients with persistent absolute lymphocytosis $\left(>5 \times 10^{9} / \mathrm{L}\right)$ for at least 3 months period with characteristic immunophenotype of mature B cell neoplasm with CD5, CD19 expression, restricted kappa or lambda light chain expression, CD5/CD19 co-expression, CD25, C103, and/or CD11c expression 
(Moreau et al 1997)

\section{Methods}

The diagnosis of mature B-cell neoplasm was established according to the WHO classification based on clinical data, and on morphologic, immunophenotypic, and genetic criteria whenever possible (Matutes and Polliack, 2000). All patients were subjected to complete history taking and Clinical examination.

Laboratory investigations included Complete blood picture using cell counter (Cell DYN), Routine Bone marrow examination (BME) by both bone marrow aspiration and biopsy sampling. Routine Immunophenotyping of whole Peripheral blood (PB) or BM using flow cytometer (Coulter EPICS-XL flowcytometer, USA) was performed at the bone marrow transplantation laboratory unit, NCI, Cairo University. The following panel of monoclonal antibodies was used: (lymphoproliferative diseases panel ) CD5, CD19, CD20, CD22, CD23, CD79b, FMC7, CD10, CD38, ,CD3, CD4,CD8, CD25,103,CD11c as well as $\kappa$ and $\lambda$ light chains labeled with either fluorescinisothiocyanate (FITC) or phycoerythrin (PE). The reagents used included MoAbs supplied by Coulter Electronics for The lymphoproliferative diseases panel, Monoclonal antibody (anti CD200) supplied by Becton Dickinson Biosciences (BDB),San José,CA, USA phycoerythrin (PE) mouse monoclonal anti-human CD200. The mean percentage of CD200 positive cells was measured.

Samples were considered positive for a marker if $\geq 20 \%$ of cells expressed that marker, except for CD38 positivity was considered $\geq 30 \%$ (Matutes and Polliack 2000).

The diagnosis of MCL was confirmed by immunohistochemical detection of cyclin D1 in tissue biopsies or detection of $\mathrm{t}(11 ; 14)(\mathrm{q} 13 ; \mathrm{q} 32)$ by FISH or PCR.

Patients were followed up for at least 6 months. The Clinical Response to Treatment was monitored according to the criteria of response by the National Comprehensive Cancer Network (NCCN) (National Comprehensive Cancer Network 2016), and the European Society of Medical Oncology (ESMO) (Eichhorst et al., 2015)

\section{Sample Collection}

Two $\mathrm{ml}$ of PB collected on K-ethylene di amine tetra-acetic acid (K-EDTA) for CBC and preparation of Leishman-stained PB smears. One ml PB or BM sample on EDTA for immunophenotyping and flow cytometric detection of CD200. Bone marrow aspirates or PB samples were processed within 24 hours of collection, being preserved at room temperature.

\section{Statistical Methods}

Data was analyzed using IBM SPSS advanced statistics version 22 (SPSS Inc., Chicago, IL). Numerical data were expressed as mean and standard deviation or median and range as appropriate. Qualitative data were expressed as frequency and percentage. Chi-square test or Fisher's exact test was used to examine the relation between qualitative variables. For not normally distributed quantitative data, comparison between two groups was done using Mann-Whitney test (non-parametric t-test). Comparison between 3 groups was done using Kruskal-Wallis test (non-parametric ANOVA) then post-Hoc "Schefe test" was used for pair-wise comparison based on Kruskal-Wallis distribution. Spearman-rho method was used to test correlation between numerical variables. All tests were two-tailed. A p-value $\leq 0.05$ was considered significant.

\section{Results}

The Present study included consecutive 67 Patients newly diagnosed Mature B- Cell neoplasms (MBN) who presented to the outpatient Clinic of National Cancer Institute, Cairo University during the period from March 2014 to December 2014. Another 20 healthy age matched normal volunteers as control subjects were also studied. All patients were followed up for a minimum period of 6 months after receiving medical treatment.

The patients included were CLL $(43,64.2 \%)$, MCL $(5,7.5 \%), \operatorname{HCL}(9,13.4 \%)$ and others $(10,15 \%)$. The later included Low grade Non-Hodgkin Lymphoma (3, 4.5\%), Mature B-Cell NHL with kappa light Chain restriction $(4,6 \%)$, Mature B-Cell NHL with lambda light chain restriction (1, 1.5\%), Marginal Zone Lymphoma (1, 1.5\%) Wandwaldenström's Macroglobulinemia $(1,1.5 \%)$ of the total studied group for each. They were classified into 4 major groups: CLL, MCL, HCL and the last 5 groups were grouped together as Others for the purpose of statistical analysis. Clinical and hematological data of the different studied groups are presented in (Table 1 and 2).

Treatment received depended on the diagnosis. CLL patients received several regimens. This included CVP (9/43, 20.9\%), Fludarabine/cyclophosphamide (11/43, 25.6\%), leukeran / steroids $(14 / 43,32.6 \%)$. A minority were kept under follow up $(9 / 43,20.9 \%)$. All Hairy cell leukemia $(9 / 9,100 \%)$ received Cladribine. Other lymphoproliferative disorders received either CVP $(3 / 15,20 \%)$ or CHOP like regimen $(12 / 15,80 \%)$.

\section{CD200 expression in the different disease groups}

All CLL and HCL patients showed positive CD200 expression (Figure.1aandb) whereas all MCL patients were negative for CD 200 (Figure 2). Among other groups of Non-Hodgkin Lymphoma, three patients (30\%) were positive for CD200 (one patient with Low grade NHL, and two patients with mature B-Cell NHL with $\kappa$ chain restriction).

The mean CD 200 Expression was $78.9 \%( \pm 20.74)$ in CLL, $2.65 \%( \pm 4.20)$ in MCL , $39.6( \pm 23.55)$ in HCL and $21.9 \%( \pm 33.8)$ in other NHL group. There was a high statistical significant difference between the CLL patients and other types of NHL ( $p<0.001)$. The difference was between CLL on one side and MCL and NHL on the other side; no significant difference was encountered between the other groups.

\section{Correlation Studies}

Correlation study was done between the expression CD 

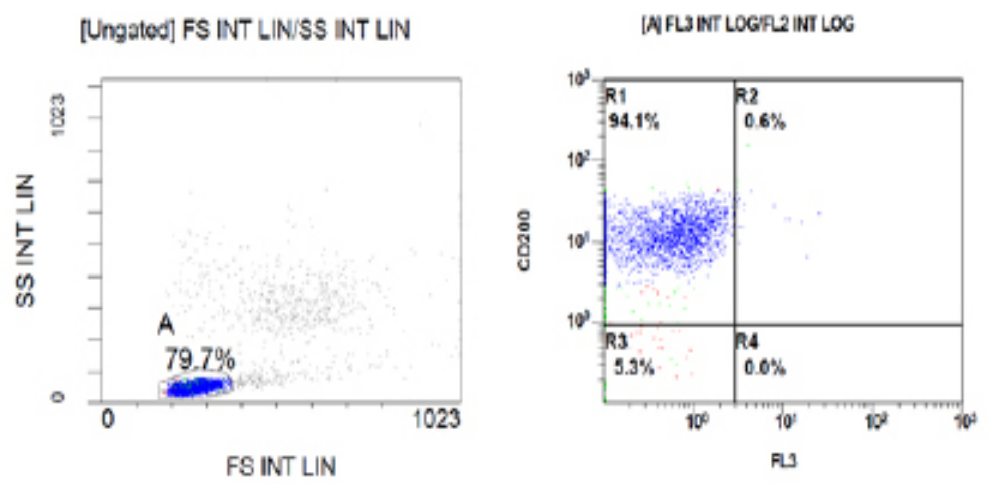

Figure 1a. CD200 Positive Expression in a CLL Case (Score5)
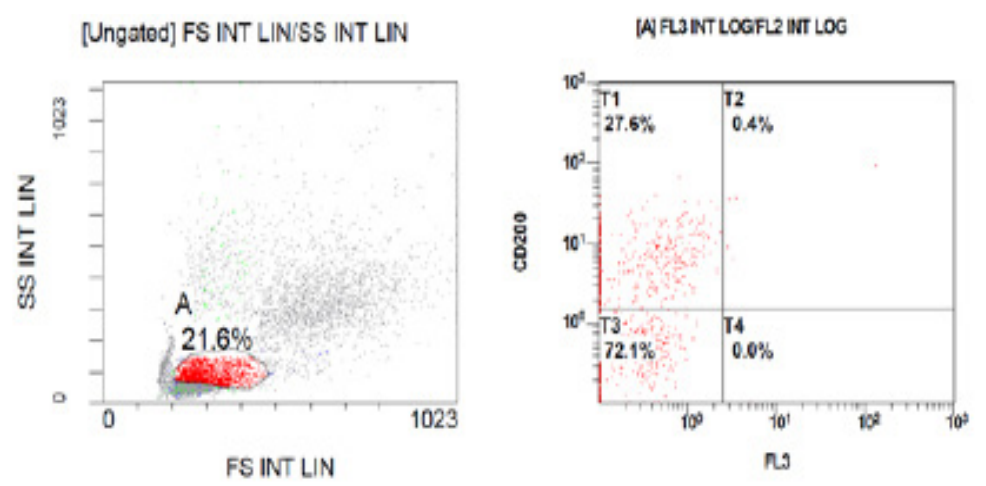

Figure 1b. CD200 Positive Expression in a HCL Case
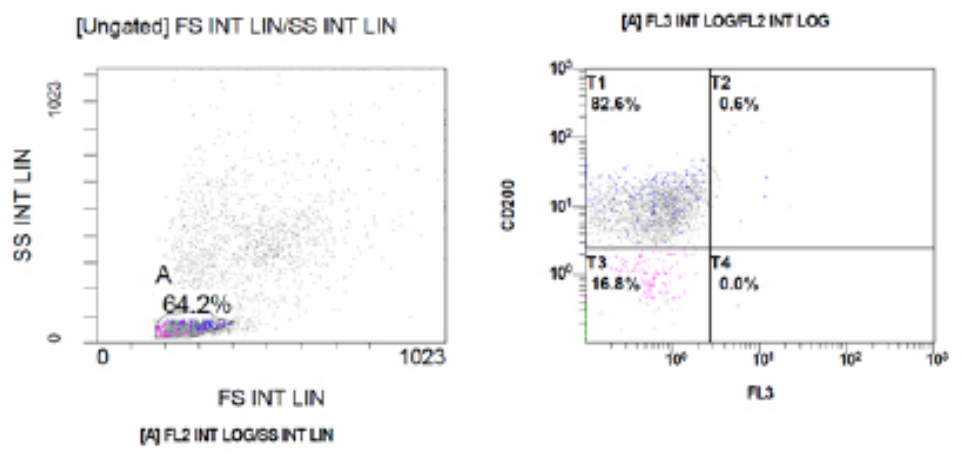

Figure 1c. CD200 Positive Expression Atypical CLL Case (score 3)

200 and various Hematological and immunophenotyping data of patients. There was a fair positive correlation between the expression of CD 200 and total Leucocytic count with( $\mathrm{r}=0.512, \mathrm{p}<0.001)$, and between the expression of CD200 and percent of peripheral blood lymphocytes $(\mathrm{r}=0.405, \mathrm{p}=0.001)$. A week positive correlation between the expression of CD 200 and hemoglobin percent, CD5 expression and CD5, 19 coexpression with $(\mathrm{r}=0.248, \mathrm{p}=0.046),(\mathrm{r}=0.282, \mathrm{p}$ $=0.021),(\mathrm{r}=0.341, \mathrm{p}=0.006)$ respectively. A good positive correlation was found between the expression of CD 200 and CD 23 expression with $(r=0.646, p<0.001)$.

Comparison between CD 200 positive and negative groups

CD 200 positivity was significantly correlated with splenomegaly $(\mathrm{P}=0.011)$, anemia $(\mathrm{p}=0.023)$ and $\mathrm{CD} 23 \%$ $(\mathrm{P}=<0.001)$. There was borderline relation to male gender $(\mathrm{P}=0.054)$. The response to treatment was more evident in CD200 expression (36/42, 66.7\% vs 6/13, $46.2 \%$ ) however this did not reach statistical significance $(\mathrm{p}=0.169)$ (Table 3 and 4).

The relation of treatment response and CD 200 expression

Positive CD 200 expression in the study group was associated with $66.7 \%(36 / 54)$ of complete or partial remission and 46.2\% (6/13) with Stationary or Progressive disease, with no significant difference between the two groups. The mean CD200 was $58.5 \pm 36.2$ and $50.5 \pm 39.3$ respectively with no significant statistical difference. Among CLL patients 68.4\% (26/43) showed complete or partial response to treatment while $31.6 \%(12 / 43)$ showed no response. No patient with MCL showed a response to treatment. The majority of $\operatorname{HCL}(75 \%, 6 / 9)$ responded to treatment while $25 \%(3 / 9)$ did not respond. Most of the other B-Cell NHL responded to treatment $(70 \%, 7 / 10)$ patients while $30 \%(3 / 10)$ showed no response in the form of stationary or progressive disease (Table 2).

CLL with unusual immunophenotyping (IMP)

CLL patients showed unusual IMP in 51.16\% (22/43) of cases and all were positive for CD200. $\mathrm{CD} 79 \mathrm{~b}+$ positive was present in $34.88 \%(15 / 43)$ of 
Table 1.Clinical Characteristics of the Patients

\begin{tabular}{|c|c|c|c|c|}
\hline Parameter Frequency (\%) & $\operatorname{CLL}(n=43)$ & $\operatorname{MCL}(\mathrm{n}=5)$ & $\operatorname{HCL}(\mathrm{n}=9)$ & Others $(\mathrm{n}=10)$ \\
\hline \multicolumn{5}{|l|}{ Age (years): } \\
\hline Mean \pm SD : & $60.5 \pm 10.3$ & $55 \pm 14.3$ & $49.7 \pm 6.2$ & $55.8 \pm 12.6$ \\
\hline Median (Range): & $61(30-82)$ & $63(32-66)$ & $50(42-60)$ & $57.5(40-76.0)$ \\
\hline \multicolumn{5}{|l|}{ Sex: } \\
\hline Male & $26(60.5 \%)$ & $2(40.0 \%)$ & $9(100.0 \%)$ & $2(20.0 \%)$ \\
\hline Female & $17(39.5 \%)$ & $3(60 \%)$ & & $8(80.8 \%)$ \\
\hline \multicolumn{5}{|l|}{ Lymphadenopathy: } \\
\hline Present: & $37(86 \%)$ & $5(100.0 \%)$ & $5(55.6 \%)$ & $6(60 \%)$ \\
\hline Absent: & $6(14 \%)$ & & $4(44.4 \%)$ & $4(40 \%)$ \\
\hline \multicolumn{5}{|l|}{ Splenomegaly: } \\
\hline Present: & $23(53.5 \%)$ & $5(100 \%)$ & $9(100 \%)$ & $8(80 \%)$ \\
\hline Absent: & $18(46.5 \%)$ & $0(0 \%)$ & $0(0 \%)$ & $2(20 \%)$ \\
\hline \multicolumn{5}{|l|}{ Hepatomegaly } \\
\hline Present: & $22(51.2 \%)$ & $3(60.0 \%)$ & $4(44.4 \%)$ & $7(70 \%)$ \\
\hline Absent: & $21(48.8 \%)$ & $2(40.0 \%)$ & $5(55.6 \%)$ & $3(30 \%)$ \\
\hline \multicolumn{5}{|l|}{ Anemia :( $\mathrm{Hb}<11 \mathrm{gm} / \mathrm{dl}$.): } \\
\hline Present: & $17(39.5 \%)$ & $3(60.0 \%)$ & $7(77.8 \%)$ & $1(10 \%)$ \\
\hline Absent: & $26(60.5 \%)$ & $2(40.0 \%)$ & $2(22.2 \%)$ & $9(90 \%)$ \\
\hline \multicolumn{5}{|l|}{ Thrombocytopenia : } \\
\hline \multicolumn{5}{|l|}{$(\mathrm{PLT}<100,000)$} \\
\hline Present: & $3(7 \%)$ & $2(40.0 \%)$ & $5(55.6 \%)$ & $2(20 \%)$ \\
\hline Absent: & $40(93 \%)$ & $3(60.0 \%)$ & $4(44.4 \%)$ & $8(80 \%)$ \\
\hline \multicolumn{5}{|l|}{ Absolute Lymphocytosis : } \\
\hline$\geq 4000 / \mu \mathrm{l}$ & $41(95.3 \%)$ & $3(60.0 \%)$ & $2(22.2 \%)$ & $8(80 \%)$ \\
\hline$<4000 / \mu l$ & $2(4.7 \%)$ & $2(40.0 \%)$ & $7(77.8 \%)$ & $2(20 \%)$ \\
\hline \multicolumn{5}{|l|}{ Bone Marrow Cellularity: } \\
\hline Normocellular: & $8(17.5 \%)$ & $1(20 \%)$ & $4(44.4 \%)$ & $4(40 \%)$ \\
\hline Hypercellular: & $34(80.0 \%)$ & $3(57.1 \%)$ & $1(11.1 \%)$ & $5(50 \%)$ \\
\hline Hypocellular: & $1(2.5 \%)$ & $1(20 \%)$ & $4(44.4 \%)$ & $1(10 \%)$ \\
\hline Total: & 43 & 5 & 9 & 10 \\
\hline \multicolumn{5}{|l|}{ Bone marrow lymphocytes: } \\
\hline$\geq 20 \%$ & $42(97.7 \%)$ & $4(80 \%)$ & $9(100 \%)$ & $8(80 \%)$ \\
\hline$<20 \%$ : & $1(2.3 \%)$ & $1(20 \%)$ & $0(0 \%)$ & $2(20 \%)$ \\
\hline Total: & 43 & 5 & 9 & 10 \\
\hline \multicolumn{5}{|l|}{ Rai Staging of CLL Patients : } \\
\hline 0 & $0(0 \%)$ & & & \\
\hline I & $12(27.9 \%)$ & & & \\
\hline II & $14(22.6 \%)$ & & & \\
\hline III and IV. & $17(39.5 \%)$ & & & \\
\hline Total: & 43 & & & \\
\hline \multicolumn{5}{|c|}{ Binet Staging of CLL patients: } \\
\hline A & $0(0 \%)$ & & & \\
\hline B & $25(58.1 \%)$ & & & \\
\hline $\mathrm{C}$ & $18(41.9 \%)$ & & & \\
\hline Total: & 43 & & & \\
\hline \multicolumn{5}{|l|}{ Hairy Cell leukemia Staging: } \\
\hline $\mathrm{I}, \mathrm{II}$ & & & 0 & \\
\hline III & & & $9(100 \%)$ & \\
\hline \multicolumn{5}{|l|}{ Lymphoma Staging: } \\
\hline I,II, III & & 0 & & 0 \\
\hline IV & & $5(100 \%)$ & & $10(100 \%)$ \\
\hline \multicolumn{5}{|l|}{ Response to treatment } \\
\hline $\mathrm{CR}+\mathrm{PR}$ & $29(67.4 \%)$ & 0 & $6(66.6 \%)$ & $7(70 \%)$ \\
\hline $\mathrm{SD}+\mathrm{PD}$ & $14(32.6 \%)$ & $5(100 \%)$ & $3(33.3 \%)$ & $3(30 \%)$ \\
\hline
\end{tabular}

3386 Asian Pacific Journal of Cancer Prevention, Vol 18 
DOI:10.31557/APJCP.2018.19.12.3383

CD200 in Mature B Cell Lymphophoproliferative Neoplasms

Table 2. Hematological and Laboratory Characteristics of the Patients

\begin{tabular}{|c|c|c|c|c|}
\hline Parameter Frequency (\%) & $\operatorname{CLL}(\mathrm{n}=43)$ & $\operatorname{MCL}(n=5)$ & $\mathrm{HCL}(\mathrm{n}=9)$ & Others $(n=10)$ \\
\hline \multicolumn{5}{|l|}{ TLC $\left(\times 10^{3} / \mu \mathrm{L}.\right)$} \\
\hline Mean \pm SD & $158.6 \pm 171$ & $126.8 \pm 258.6$ & $4.3 \pm 2.9$ & $41.6 \pm 47$ \\
\hline Median (Range). & $93.9(7.1-93.1)$ & $12.3(3.9-89.3)$ & $2.4(1.5-9.5)$ & $16.2(4.2-48.2)$ \\
\hline \multicolumn{5}{|l|}{$\mathrm{Hb}(\mathrm{gm} / \mathrm{dL})}$. \\
\hline Mean $\pm \mathrm{SD}$ & $10.9 \pm 2.7$ & $9.6 \pm 4.3$ & $9.4 \pm 2.8$ & $8.4 \pm 1.7$ \\
\hline Median (Range). & $11.1(5.0-15.8)$ & $8.3(4-15)$ & $8.9(5.8-15.1)$ & $8.5(6.3-11.8)$ \\
\hline \multicolumn{5}{|l|}{$\operatorname{PLT}\left(\times 10^{3} / \mu \mathrm{L}.\right)$} \\
\hline Mean \pm SD & $182.8 \pm 86.5$ & $197.4 \pm 141.7$ & $119 \pm 101.2$ & $136.2 \pm 67.7$ \\
\hline Median (Range). & $169(10-441)$ & $133(76-410)$ & $99(20-333.0)$ & $152.5(22-232)$ \\
\hline \multicolumn{5}{|c|}{ Relative Lymphocytic Count (\%) } \\
\hline Mean \pm SD: & $84.7 \pm 13.8$ & $70 \pm 27.8$ & $62.2 \pm 22.7$ & $80.1 \pm 16$ \\
\hline Median (Range): & $90(45-100)$ & $85(38-97)$ & $65(14-90)$ & $82(48-97)$ \\
\hline \multicolumn{5}{|c|}{ Absolute Lymphocytic Count $\left(\times 10^{3} / \mu \mathrm{L}\right.$. $)$} \\
\hline Mean \pm SD: & $148.8 \pm 169.3$ & $121.1 \pm 251.9$ & $2.4 \pm 1.8$ & $37.5 \pm 43.4$ \\
\hline Median (Range): & $85.6(3.2-785.1)$ & $10.4(1.5-571.7)$ & $1.5(0.8-5.9)$ & $13.5(3.1-132)$ \\
\hline \multicolumn{5}{|l|}{ Marrow Lymphocytes (\%) } \\
\hline Mean $\pm \mathrm{SD}$ : & $69 \pm 19.2$ & $69.6 \pm 17.6$ & $67.8 \pm 22.8$ & $54.7 \pm 24.4$ \\
\hline Median (Range): & $70(18-98)$ & $72(51-95)$ & $70(24-95)$ & $60(18-86)$ \\
\hline \multicolumn{5}{|l|}{ CD $5(\%)$} \\
\hline Mean \pm SD: & $76.5 \pm 20.9$ & $74 \pm 18$ & $10.4 \pm 13.3$ & $52.2 \pm 28.5$ \\
\hline Median (Range) : & $80(24.6-100)$ & $76.5(48-94.4)$ & $2.6(0.2-33)$ & $57.5(7-88)$ \\
\hline \multicolumn{5}{|c|}{ CD 5, 19 (\%) Co-expression: } \\
\hline Mean \pm SD: & $68.8 \pm 22.3$ & $64.7 \pm 22.8$ & $1.2 \pm 1.7$ & $41.2 \pm 27.7$ \\
\hline Median (Range): & $75(2.9-93.1)$ & $67(35.7-88)$ & $0.1(0.0-3.4)$ & $42.1(0.2-80.1)$ \\
\hline \multicolumn{5}{|l|}{ CD $23(\%)$} \\
\hline Mean \pm SD: & $61.4 \pm 24.5$ & $15.4 \pm 30$ & $1.5 \pm 1.9$ & $5.3 \pm 12.5$ \\
\hline Median (Range): & $66.1(0.2-96.5)$ & $2.5(0.4-69.0)$ & $1.1(0.0-4.3)$ & $0.4(0.1-40.1)$ \\
\hline \multicolumn{5}{|l|}{ CD 38: $(\%)$} \\
\hline Mean \pm SD: & $22.1 \pm 26.1$ & $47 \pm 29.7$ & $19.4 \pm 26.4$ & $43.6 \pm 32$ \\
\hline Median (Range): & $14(0.2-82)$ & $47(26-68)$ & $19.4(0.7-38)$ & $51.6(8.4-70.8)$ \\
\hline Valid No: & 12 & 2 & 2 & 3 \\
\hline \multicolumn{5}{|l|}{ CD $103(\%)$} \\
\hline Mean \pm SD: & & & $62.1 \pm 25.2$ & \\
\hline Median (Range): & & & $63(22-88)$ & \\
\hline \multicolumn{5}{|l|}{ CD $25(\%)$} \\
\hline Mean \pm SD: & & & $54.4 \pm 30.7$ & \\
\hline Median (Range): & & & 44 (20-91.9) & \\
\hline \multicolumn{5}{|l|}{ CD11c: (\%) } \\
\hline Mean \pm SD: & & & $46 \pm 11.6$ & \\
\hline Median (Range): & & & $46(30.9-70)$ & \\
\hline \multicolumn{5}{|l|}{ CD 200: (\%) } \\
\hline Mean & 78.9 & 2.65 & 39.69 & 21.9 \\
\hline SD & 20.74 & 4.20 & 23.55 & 33.8 \\
\hline Median & 87 & 1.32 & 30.5 & 1.4 \\
\hline Range & $(20-98)$ & $(0.04-10.10)$ & $(20-71.4)$ & $(0.5-83.6)$ \\
\hline
\end{tabular}

*TLC, Total leukocytic count; Hb, Hemoglobin; PLT, Platelets; St, Stage; I, Stage 1; II, Stage 2; III, Stage 3; IV, Stage 4; CR, Complete remission; PR, Partial remission; SD, Stationary Disease; PD, progressive Disease; *Samples were considered positive for a marker if $\geq 20 \%$ of cells expressed that marker, except for CD38 positivity was considered $\geq 30 \%$.(Matutes and Polliack, 2000). 
Table 3. Relation between CD200 Expression and Different Clinical Characteristics in 67 Mature B Nell Lymphophoproliferative Neoplasms

\begin{tabular}{|c|c|c|c|c|}
\hline \multirow[t]{2}{*}{ Parameter Frequency (\%) } & \multicolumn{2}{|c|}{ CD200 } & \multirow[t]{2}{*}{ Total } & \multirow[t]{2}{*}{ P Value } \\
\hline & Positive expression $(n=55)$ & Negative expression $(n=12)$ & & \\
\hline \multicolumn{5}{|l|}{ Age (years) } \\
\hline Mean \pm SD & $58.5 \pm 10.5$ & $55 \pm 13$ & & 0.422 \\
\hline Median (Range) & $60(30-82)$ & $57.5(32-76)$ & & \\
\hline \multicolumn{5}{|l|}{ Sex } \\
\hline Male & $35(89.7 \%)$ & $4(10.3 \%)$ & 39 & \\
\hline Female & $20(71.4 \%)$ & $8(28.6 \%)$ & 28 & 0.054 \\
\hline Total & $55(82.1 \%)$ & $12(17.9 \%)$ & 67 & \\
\hline \multicolumn{5}{|l|}{ Lymphadenopathy } \\
\hline Present & $43(81.1 \%)$ & $10(8.9 \%)$ & 53 & \\
\hline Absent & $12(85.7 \%)$ & $2(14.3 \%)$ & 14 & 0.749 \\
\hline Total & $55(82 \%)$ & $12(18 \%)$ & 67 & \\
\hline \multicolumn{5}{|l|}{ Splenomegaly } \\
\hline Present & $33(73.3 \%)$ & $12(26.7 \%)$ & 45 & \\
\hline Absent & $22(100 \%)$ & $0(0 \%)$ & 22 & 0.011 \\
\hline Total & $55(82 \%)$ & $12(18 \%)$ & 67 & \\
\hline \multicolumn{5}{|l|}{ Hepatomegaly } \\
\hline Present & $27(75 \%)$ & $9(25 \%)$ & 36 & \\
\hline Absent & $27(90 \%)$ & $3(10 \%)$ & 30 & 0.104 \\
\hline Total & $54(80.6 \%)$ & $12(19.4 \%)$ & 67 & \\
\hline \multicolumn{5}{|c|}{ Rai Staging system of CLL patients:( $n=43)$} \\
\hline 0 & 0 & 0 & 0 & \\
\hline I & $12(100 \%)$ & $0(0 \%)$ & 12 & \\
\hline II & $14(100 \%)$ & $0(0 \%)$ & 18 & \\
\hline III and IV & $15(88.2 \%)$ & $2(11.8 \%)$ & 18 & 0.236 \\
\hline Total: & $41(95.4 \%)$ & $2(4.6 \%)$ & 43 & \\
\hline \multicolumn{5}{|c|}{ Binet Staging of CLL patients:(n=43) } \\
\hline A & 0 & 0 & 0 & \\
\hline $\mathrm{B}$ & $25(100 \%)$ & $0(0 \%)$ & 25 & \\
\hline $\mathrm{C}$ & $16(88.9 \%)$ & $2(11.1 \%)$ & 18 & 0.094 \\
\hline Total: & $41(95.4 \%)$ & $2(4.6 \%)$ & 43 & \\
\hline \multicolumn{5}{|l|}{ Response to Treatment: } \\
\hline CR+PR: & $36(85.7 \%)$ & $6(14.3 \%)$ & 42 & 0.169 \\
\hline SD+PD: & $18(72 \%)$ & $7(28 \%)$ & 25 & \\
\hline Total: & $54(80.6 \%)$ & $13(19.4 \%)$ & 67 & \\
\hline
\end{tabular}

CR, Complete Remission; PR, Partial Remission; SD, Stationary Disease; PD, Progressive Disease; *Samples were considered positive for a marker if $\geq 20 \%$ of cells expressed that marker, except for CD38 positivity was considered $\geq 30 \%$ (Matutes and Polliack, 2000).

cases, CD23- negative in 3 cases (6.98\%) and two (4.65\%) of them were atypical CLL cases (score 3 on the Matutes scoring system)

CD22 bright B was present in one case $(2.33 \%)$, FMC7+ positive in 4 cases $(9.3 \%)$ and bright Surface membrane immunoglobulin (Sm Ig ) in one case (2.33\%). There were three cases among the above showing 2 unusual IMP. CD79b + was associated with FMC7+ in one case. The other 2 cases had CD23-negative, one combined with CD22 bright and the other with bright Sm Ig.

\section{Response to treatment and different prognostic factors}

Analysis of the response to treatment and different prognostic factors was done in the whole group (67 patients). This included clinical, hematological and immunophenotyping. They did not show any significant statiscal difference within any of them.

High CD $200 \geq 50 \%$

In the CLL group, comparison of high $(\geq 50 \%)$ and low $(<50 \%)$ CD200 expression was done as regards different clinical and hematological variables Table 3. The CD $200 \geq 50 \%$ had a higher mean age $(60.8 \pm 10.7$ vs $57.5 \pm 3.3)$, mean total leucocytic count ( $172 \pm 174.4$ vs $31.7 \pm 30.2)$, mean platelet count $(86.8 \pm 12.6$ vs $64.8 \pm 7.4)$ and mean peripheral absolute lymphocytic count (162.45 \pm 172.67 vs $22.131 \pm 23.740)$. Also the expression of mean CD5, 
DOI:10.31557/APJCP.2018.19.12.3383

CD200 in Mature B Cell Lymphophoproliferative Neoplasms

Table 4. Relation Between CD200 Expression and Hematological Characteristics in 67 Mature B Cell Lymphophoproliferative Neoplasms

\begin{tabular}{|c|c|c|c|c|}
\hline \multirow[t]{2}{*}{ Parameter Frequency (\%) } & \multicolumn{2}{|c|}{ CD200 } & \multirow[t]{2}{*}{ Total } & \multirow[t]{2}{*}{ P-Value } \\
\hline & Positive expression $(\mathrm{n}=55)$ & Negative expression $(n=12)$ & & \\
\hline \multicolumn{5}{|l|}{ Anemia $<11 \mathrm{gm} / \mathrm{dl}$} \\
\hline Present: & $25(89.3 \%)$ & $3(10.7 \%)$ & 28 & \\
\hline Absent: & $28(71.8 \%)$ & $11(28.2 \%)$ & 39 & 0.023 \\
\hline Total: & $53(79.1 \%)$ & $14(20.8 \%)$ & 67 & \\
\hline \multicolumn{5}{|c|}{ Thrombocytopenia < 100,000/ul.) } \\
\hline Present: & $9(75 \%)$ & $3(25 \%)$ & 12 & \\
\hline Absent: & $46(83.6 \%)$ & $9(16.4 \%)$ & 55 & \\
\hline Total: & $55(82 \%)$ & $12(18 \%)$ & 67 & 0.673 \\
\hline \multicolumn{5}{|l|}{ Bone Marrow Cellularity: } \\
\hline Normocellular: & $14(82.4 \%)$ & $3(17.6 \%)$ & 17 & \\
\hline Hypercellular: & $36(83.7 \%)$ & $7(16.3 \%)$ & 43 & \\
\hline Hypocellular: & $6(85.7 \%)$ & $1(14.3 \%)$ & 7 & \\
\hline Total: & $54(80.6 \%)$ & $13(19.4 \%)$ & 67 & 1.000 \\
\hline \multicolumn{5}{|l|}{ Bone Marrow Lymph. } \\
\hline$>=20 \%$ & $52(82.5 \%)$ & $11(17.5 \%)$ & 63 & 0.321 \\
\hline$<20 \%$ & $2(50 \%)$ & $2(50 \%)$ & 4 & \\
\hline Total: & $54(80.6 \%)$ & $13(19.4 \%)$ & 67 & \\
\hline \multicolumn{5}{|l|}{$\operatorname{TLC}\left(\times 10^{3} / \mu \mathrm{L}.\right)$} \\
\hline Mean $\pm \mathrm{SD}$ : & $125.8 \pm 163$ & $79.8 \pm 166$ & & \\
\hline Median (Range): & $74(1.5-793.1)$ & $14(3.9-589.3)$ & & 0.135 \\
\hline \multicolumn{5}{|l|}{$\mathrm{Hb}(\mathrm{gm} / \mathrm{dL})}$. \\
\hline Mean \pm SD: & $10.5 \pm 2.7$ & $8.6 \pm 3$ & & \\
\hline Median (Range): & $10.8(5-15.8)$ & $8.4(4-15)$ & & 0.027 \\
\hline \multicolumn{5}{|l|}{$\operatorname{PLT}\left(\times 10^{3} / \mu \mathrm{L}.\right)$} \\
\hline Mean \pm SD: & $169.4 \pm 91.6$ & $161.8 \pm 100.4$ & & \\
\hline Median (Range): & $150(10-441)$ & $151.5(22-410)$ & & 0.624 \\
\hline \multicolumn{5}{|l|}{ PB Lymphocytes(\%) } \\
\hline Mean \pm SD: & $80.9 \pm 17.4$ & $74.6 \pm 21.7$ & & \\
\hline Median (Range): & $88(14-100)$ & $80(38-97)$ & & 0.326 \\
\hline \multicolumn{5}{|c|}{ Absolute lymph.count: $\left(\times 10^{3} / \mu \mathrm{L}.\right)$} \\
\hline Mean $\pm \mathrm{SD}$ : & $117.1 \pm 160$. & $74.6 \pm 161.5$ & & \\
\hline Median (Range): & $57.5(0.785-785.1)$ & $111.16(1.47-571.650$ & & 0.166 \\
\hline \multicolumn{5}{|c|}{ Bone Marrow Lymphocytes(\%) } \\
\hline Mean \pm SD: & $69.2 \pm 19.2$ & $55.7 \pm 23.7$ & & 0.094 \\
\hline Median (Range): & $70.5(18-98)$ & $55(18-95)$ & & \\
\hline \multicolumn{5}{|l|}{ CD5 (\%) } \\
\hline Mean \pm SD: & $65.4 \pm 31.3$ & $56.3 \pm 29.1$ & & 0.239 \\
\hline Median (Range): & $75(0.2-100)$ & $57.5(7-94.6)$ & & \\
\hline \multicolumn{5}{|l|}{ CD 5,19 coexpression (\%) } \\
\hline Mean \pm SD: & $61.3 \pm 29.3$ & $47.7 \pm 29.3$ & & 0.102 \\
\hline Median (Range): & $73.2(0.01-93.1)$ & $50.1(0.2-88)$ & & \\
\hline \multicolumn{5}{|l|}{ CD 23(\%) } \\
\hline Mean \pm SD: & $50.2 \pm 32.2$ & $10.1 \pm 21.7$ & & \\
\hline Median (Range): & $57(0.01-96.5)$ & $1.4(0.1-69)$ & & $<0.001$ \\
\hline CD $38(\%)$ & $(\mathrm{n}=15)$ & $(n=4)$ & & \\
\hline Mean \pm SD: & $25 \pm 27.3$ & $38.5 \pm 26.5$ & & \\
\hline Median (Range): & $16(0.2-82)$ & $38.8(8.4-68)$ & & 0.230 \\
\hline
\end{tabular}


Table 5. CD200 Expression Above and Below 50\% in the CLL Group

\begin{tabular}{|c|c|c|c|}
\hline \multirow[t]{2}{*}{ Parameter } & \multicolumn{2}{|c|}{ CD200 } & \multirow[t]{2}{*}{$\mathrm{P}$ value } \\
\hline & $\geq 50 \%$ (high expression) $(\mathrm{n}=31)$ & $<50 \%$ (low expression) $(n=12)$ & \\
\hline \multicolumn{4}{|l|}{ Sex } \\
\hline Male & $19(73.08 \%)$ & $7(26.92 \%)$ & \\
\hline Female & $12(70.59 \%)$ & $5(29.41 \%)$ & $<0.001$ \\
\hline \multicolumn{4}{|l|}{ Hb:(gm/dL.): } \\
\hline$\geq 11 \mathrm{gm} / \mathrm{dL}$ & $17(65.38 \%)$ & $9(34.62 \%)$ & \\
\hline$<11 \mathrm{gm} / \mathrm{dL}$ & $14(82.35 \%)$ & $3(17.65 \%)$ & 0.15 \\
\hline \multicolumn{4}{|l|}{ Platelets $\left(\times 10^{3} / \mu \mathrm{L}.\right)$ : } \\
\hline$\geq 100$ & $29(72.5 \%)$ & $11(27.5 \%)$ & \\
\hline$<100$ & $2(66.67 \%)$ & $1(33.33 \%)$ & 0.3 \\
\hline Lymphadenopathy, No. (\%): & $26(70.27 \%)$ & $11(29.73 \%)$ & 0.15 \\
\hline Splenomegaly, No. (\%): & $20(86.96 \%)$ & $3(13.04 \%)$ & 0.02 \\
\hline Hepatomegaly, No. (\%): & $18(85.71 \%)$ & $3(14.29 \%)$ & 0.05 \\
\hline \multicolumn{4}{|l|}{ Rai staging system } \\
\hline Stage I & $3(25 \%)$ & $9(75 \%)$ & 0.05 \\
\hline Stage II & $13(92.86 \%)$ & $1(7.14 \%)$ & \\
\hline Satge III and IV & $15(89.12 \%)$ & $2(11.76 \%)$ & \\
\hline \multicolumn{4}{|l|}{ Binet staging system: } \\
\hline Stage A & 0 & 0 & \\
\hline B & $15(60 \%)$ & $10(40 \%)$ & \\
\hline $\mathrm{C}$ & $16(88.89 \%)$ & $2(11.11 \%)$ & 0.003 \\
\hline \multicolumn{4}{|l|}{ Response to treatment } \\
\hline $\mathrm{CR}+\mathrm{PR}$ & $20(68.97 \%)$ & $9(31.03 \%)$ & \\
\hline $\mathrm{SD}+\mathrm{PD}$ & $11(78.57 \%)$ & $3(21.43 \%)$ & 0.45 \\
\hline Time to treatment in month & 3 & 29 & 0.0007 \\
\hline Overall survival & $81 \%$ & $83 \%$ & 0.034 \\
\hline
\end{tabular}

CD19coexpession ( $70.8 \pm 21$ vs $49.3 \pm 28.9)$ as well as the CD 23 were higher $(62.8 \pm 22.8$ vs $47.6 \pm 38.7)$. Table 5 .

The high CD 200 group had significant male predominance, splenomegaly and hepatomegaly $(p=>0.001,0.02,0.05$ respectively). They also had more advanced stage in both the Rai and the Binet staging system $(\mathrm{p}=0.05$ and 0.003$)$. The initial response to treatment was good but was not significant. $(\mathrm{p}=0.45)$. After a median follow up of 30 month, this group was associated with earlier relapse ( three vs 25 month, $p=0007$ ) and on significant difference in survival ( $81 \%$ vs $83 \%, p=0.34)$.

\section{Discussion}

In this study, we investigated whether the use of CD200 as a novel routine marker could be helpful to improve the utility of flow cytometry in differentiating between B lymphoproliferative neoplasms, particularly between CLL cases with typical or atypical immunophenotype and MCL cases and the possible prognostic significance of this marker in CLL patients and others.

CD200 expression managed to differentiate CLL cases including those with unusual immunophenotype $(22 / 43,51.2 \%)$ from MCL. It was consistently positive in all CLL and negative in all MCL. The median expression of CD200 expression in CLL and MCL was 87 and 1.3 respectively. This is consistent with other studies (Sandes et al 2013; Eldesoukey et al., 2012; Rodrigues et al 2016), supporting the important diagnostic role of CD200 especially for differentiating CLL cases with Matutes score less than or equal to 3 from MCL being an easy and not expensive method when compared to MCL confirmation by cyclin D1immunostaining on tissue biopsy, and/or detection of $\mathrm{t}(11 ; 14)$ on MCL which are expensive, time consuming and not available in all centers .

In our study, all patients with hairy cell leukemia were CD200 positive with the highest intensity of expression among all our studied cases. These findings were consistent with previous reports (Van Dongen et al., 2012; Sandes et al 2013) suggesting this over expression could play a role in HCL survival, proliferation and the inhibition of anti-tumor immune response.

The role of CD 200 in the distinction of classical HCL, HCL variant and SMZL couldn't be demonstrated as we had no case of HCL variant in our study and due to small sample size of SMZL.

Among the six patients in CD5 positive B-Cell NHL group (non-CLL and non MCL-non PLL) CD200 expression was heterogeneous. Comparing CD200 expression between MCL and CD5 positive B-Cell NHL 

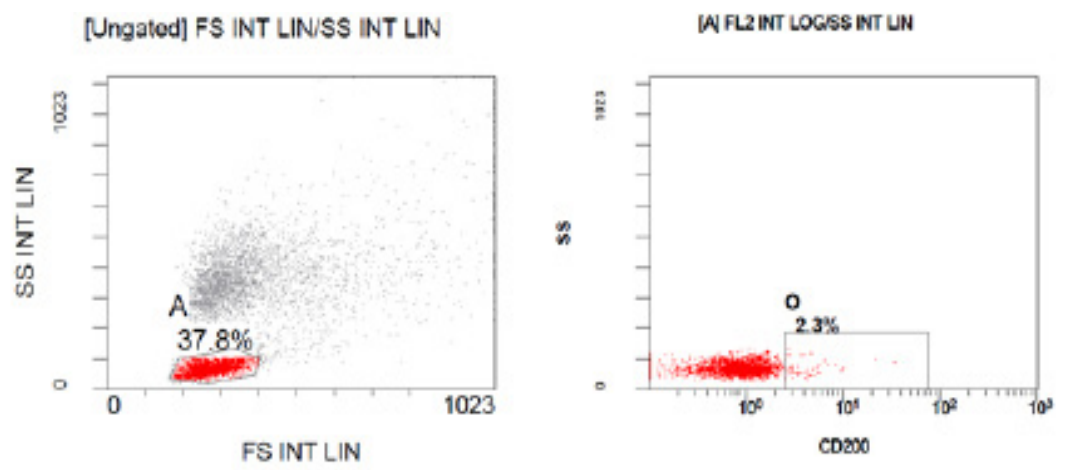

Figure 2a. CD200 Negative Expression in a Case with CD5 Positive NHL.
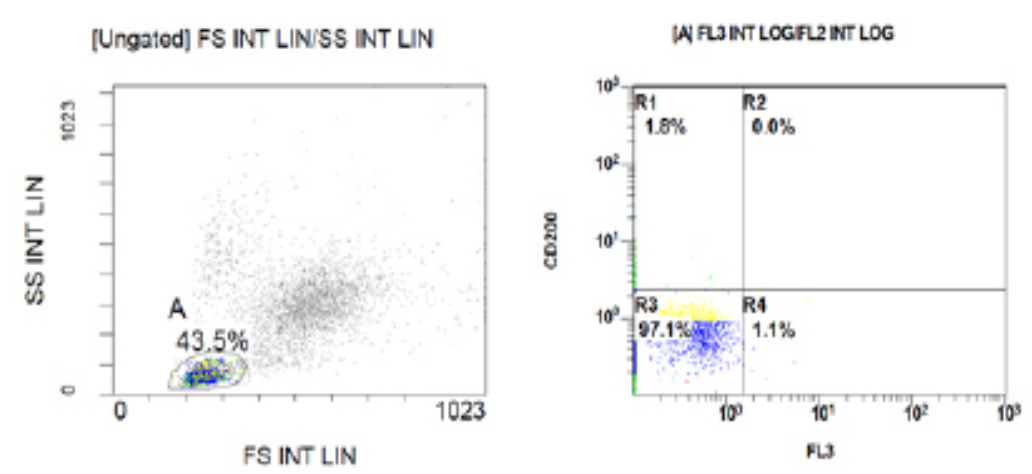

Figure 2b. CD200 Negative Expression in a MCL Case.

$\mathrm{CD} 200$ Expression in CLL patients had a mean $\pm \mathrm{SD}$ of $78.9 \pm 20.74$, In MCL a mean $\pm \mathrm{SD}$ of $2.65 \pm 4.20$, In HCL a mean $\pm \mathrm{SD}$ of $39.6 \pm 23.55$ and in other NHL group a mean \pm SD of $21.9 \pm 33.8$ with a highly significant statistical difference $(\mathrm{P}<0.001)$. This difference was observed between the CLL patients and those with MCL and other types of NHL $(p<0.001)$ while no significant difference was found between MCL patients and other NHL as regards CD200 expression.

group showed no significant difference. These results were in agreement with Sandes et al., (2013) who suggested that the absence of CD 200 in the CD5 positive NHL group cannot be considered an exclusive finding to differentiate them from MCL.

In this study CD200 expression showed good positive correlation with CD23 and weak positive correlation with CD5 and19 co-expression. There was fair correlation with the total leukocyte count and the peripheral blood lymphocytes and weak correlation with the haemoglobin . CD200 was not correlated with the clinical presentation of the patients however it showed higher percent of male gender, anemia and splenomegaly. In contrast to our results, El Desouky et al., (2012) found no correlation between CD200 expressions in other B-Cell neoplasms and clinical or laboratory data which could be attributed to larger sample size in our study.

We attempted to classify the CLL patients group based on CD200 expression into a high ( $\geq 50 \%)$ expression group and a low $(<50 \%)$ expression group and compare the clinical response between both groups. The high CD200 expression was associated with higher mean age, TLC, peripheral blood lymphocytes and absolute lymphocytic count. There was higher mean CD5, 19 coexpression and CD23 expression. It was also accompanied with significant splenomegaly, hepatomegaly ( $\mathrm{p}=0.02,0.05$ respectively). It was also associated with advanced stage on both Rai and the Binet staging system $(\mathrm{p}=0.05$ and 0.003$)$. However the initial good response was accompanied by significant shorter time to treatment but did not have a statistical impact on the overall survival. This is in accordance with Wang et al., 2014 and Wong et al., 2012 that revealed a possible impact of CD200 on prognosis and treatment in CLL patients. Also Miao et al., 2016 showed that the level of CD200 greatly affected time to progression ( two vs 28 months, $\mathrm{p}=0.0008$ ) but did not affect the overall survival $(\mathrm{p}=0.2379)$.

The study was limited by the small number of patients and short follow up. Further studies should include survival analysis to determine the proper prognosis of these patients.

We conclude that High CD200 $\geq 50$ was associated with advanced stage and earlier time to progression. The study recommends the addition of CD200 as a routine investigatory marker in the flowcytometry panel of chronic lymphoproliferative disorders to distinguish between their subtypes especially MCL and atypical CLL with unusual IMP. Blockade of CD200 should be explored in the treatment of CLL and HCL as it may improve prognosis.

\section{Disclosure and conflict of interest}

The study did not receive any fund. There was no conflict of interest.

\section{Compliance with Ethical Standards}

The study was approved by the institutional review board and the ethical committee. All the patients had to give an informed consent before being recruited.

This was in accordance with the ethical standards as laid down in the 1964 Declaration of Helsinki and its later amendments or comparable ethical standards. 


\section{Acknowledgements}

We are so grateful to the members of the NCI flowcytometry lab for their extensive cooperation in the study. Our great gratitude is to our medical colleagues and cancer registry employees for helping us to collect the cases as well as the medical data.

\section{Conflict of interest}

There was no conflict of interest.

\section{References}

Barna G, Reiniger L, Tatrai P, Kopper L, Matolcsy A( 2008). the cut off levels of CD23 expression in the differential diagnosis of MCL and CLL. Hematol Oncol, 26, 167-70.

Challagundla P, Medeiros J, Kanagal-Shamanna R, Miranda RN, Jorgensen JL (2014).Differential expression of CD200 in B-Cell neoplasms by flow cytometry can assist in diagnosis, subclassification, and bone marrow staging. Am J Clin Pathol, 142, 837-44.

Eichhorst B, Robak T, Montserrat E, et al (2015). Chronic lymphocytic leukaemia: ESMO Clinical Practice Guidelines for diagnosis, treatment and follow-up. Ann Oncol, 5, 78-84.

Eldesoukey NA, Afify RA, Amin DG, Mohammed RF (2012). CD200 expression in B cell chronic lymphoproliferative disorders. J Investig Med, 60, 56-61.

Ivancević TD, Kurtović NK, Knezević V, et al (2014). The role of immunophenotyping in differential diagnosis of chronic lymphocytic leukemia. Srp Arh Celok Lek, 142, 197-203.

Matutes E, Polliack A (2000). Morphological and immunophenotypic features of chronic lymphocytic leukemia. Rev Clin Exp Hematol, 4, 22-47.

Miao Y, Fan L, Wu Y-J, et al (2016). Low expression of CD200 predicts shorter time-to-treatment in chronic lymphocytic leukemia. Oncotarget, 7, 13551- 62.

Moreau EJ, Matutes E, A'Hern RP, et al (1997). Improvement of the chronic lymphocytic leukemia scoring system with the monoclonal antibody SN8 (cd79b). Am J Clin Pathol, 108, 378-82.

Moreaux J, Luc Veyrune J, Reme T, De Vos J, Klein B (2008). CD200: A putative therapeutic target in cancer. Biochem Biophys Res Commun, 366, 117-22.

National Comprehensive Cancer Network (2016). Non hodgkin's lymphomas version 2. NCCN. Available at http:// www.nccn.org/professionals/physician_gls/pdf/nhl.pdf. Accessed:March 11, 2016.

Rodrigues CA, Gonçalves MV, Ikoma MRV, et al (2016). Diagnosis and treatment of chronic lymphocytic leukemia: recommendations from the Brazilian Group of Chronic Lymphocytic Leukemia. Rev Bras Hematol Hemoter, 38, 346-57.

Sandes AF, Chauffaille M de L, MC Oliveira CR, et al (2013). CD200 has an important role in the differential diagnosis of mature B cell neoplasms by multiparameter flow cytometry. Cytometry B Clin Cytom, 86, 98-105.

Van Dongen JJ, Lhermitte L, Bottcher S, et al (2012). Euro flow antibody panels for standardized $n$-dimensional flow cytometric immunophenotyping of normal, reactive and malignant leukocytes. Leukemia, 26, 1908-975.

Wang X, Zhang Z, Liu Y, et al (2014). Expression of CD200 in the bone marrow of chronic lymphocytic leukemia patients and its correlations with clinical prognosis. Chin J Cell Mol Immunol, 30, 75-8.

Wong KK, Brenneman F, Chesney A, Spaner DE, Gorczynski RM (2012). Soluble CD200 is critical to engraft Chronic
Lymphocytic Leukemia cells in immunocompromised mice. Cancer Res, 72, 4931-43.

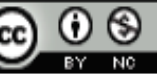

This work is licensed under a Creative Commons AttributionNon Commercial 4.0 International License. 